\title{
Skin-Layer Analysis Using Optical Coherence Tomography (OCT)
}

\author{
Eun Soo Park
}

Department of Plastic and Reconstructive Surgery, Soonchunhyang Univsersity Bucheon Hospital, Bucheon, Korea
Received June 3, 2014

Revised June 16, 2014

Accepted June 18, 2014

\section{Correspondence}

Eun Soo Park

Department of Plastic and Reconstructive Surgery, Soonchunhyang Univsersity Bucheon Hospital, 1174, Jung-dong, Wonmi-gu,

Bucheon 420-767, Korea

Tel: +82-32-621-5319

Fax: +82-32-621-5016

E-mail: peunsoodaschmc.ac.kr

(C) Korean Society for Laser Medicine and Surgery

(c) This is an open access article distributed under the terms of the Creative Commons Attribution NonCommercial License (http://creativecommons.org/ licenses/by-nc/3.0) which permits unrestricted noncommercial use, distribution, and reproduction in any medium, provided the original work is properly cited.
In the past decade, advances in optics, fiber as well as laser technology, have enabled development of a novel noninvasive optical biomedical imaging technique, optical coherence tomography (OCT). Optical coherence tomography (OCT) is a micrometer scale, noninvasive, nondestructive, cross-sectional optical imaging technique for use in biological systems. It is an interferometric technique with a low coherence light source, typically using near-infrared light, which enables deep penetration into the scattering medium without damage to the human body. It has become a prominent imaging technique for precision measurement in various applications, including diagnosis in the medical field. Investigations using OCT to assess skin structure in clinical settings started in the past decade and consequently proved that this imaging method is useful in visualizing subsurface structures of normal skin, including the epidermis, dermoepidermal junction, dermis, hair follicles, blood vessels, and sweat ducts. OCT is a promising new noninvasive realtime imaging modality for investigation of skin morphology. In some cases it may be useful for diagnosis of skin disease. Because of its noninvasive character, the technique enables monitoring of inflammatory disease over time. Objective quantification of the efficacy and tolerance of topical treatment is also possible.

\section{Key words}

Optical coherence tomography (OCT); Skin image 


\section{INTRODUCTION}

Optical coherence tomography (OCT) (Fig. 1) is a noninvasive, non-destructive technique for morphological investigation of tissue. The technique was first introduced by Fercher et al. and Huang et al. for investigation of the human eye. It has proved to be of value for visualization of changes in the retina and the cornea. In 1990, OCT is based on low-coherence interferometry (LCI) to produce depth profiles, which are equivalent to the ultrasound A-scans. In the past decade, advances in optics, fiber as well as laser technology have enabled first vitro tomogram of the human eye in 1991. 1997, for examination of a highly scattering tissue like the skin, OCT introduced in clinical field and is now increasingly employed in clinical skin research. ${ }^{1,2}$

\section{PRINCIPLES AND OPERATION}

Analogous to ultrasound imaging, OCT measures optical echo delays and the intensity of back reflected infrared light rather than acoustic waves. The OCT system is based on the more than 100 year old principle of Michelson interferometry using short coherence length light. It does not require a direct contact. In LCl, light backscattered from inside the specimen is measured by correlating it with light that has travelled a known reference path. So the principal OCT system design is an interferometer. One interferometer arm contains a modular probe that focuses and scans the light onto the sample. The second interferometer arm is the so-called

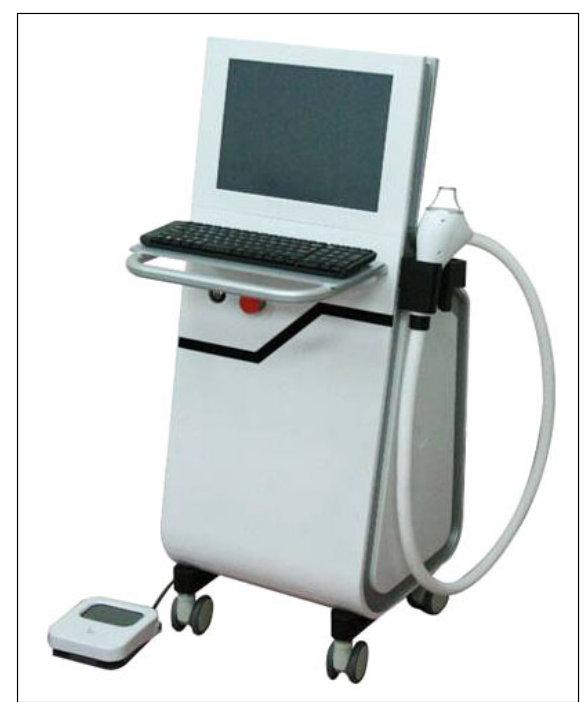

Fig. 1. The OCT system. reference path that is equipped with a mirror. The optical path length distribution of the sample beam measured by the interference modulation of the axial OCT scan can be interpreted as the depth resolved reflection signal of the sample. For OCT, light sources with wavelengths in the near infrared, most of them operating at 1,300 nm, are used. The light sources are super luminescent diodes with a short coherence length of about $15 \mathrm{~mm}$. The light is coupled to a single-mode fiberoptic interferometer and divided into a reference beam and a probe beam. The reference signal is reflected from a scanning mirror system. The light in the sample arm is focused onto the superficial skin layers, backscattered and recombined again with the reflected reference signal. ${ }^{1-4}$

In contrast to the eye, which is naturally a low light scattering transparent medium, skin is nearly nontransparent. The lack of transparency is due to absorption and scattering; the first is mainly influenced by the concentration of melanin and hemoglobin, the latter by differences in the refraction index. In the wavelength range of 700 to $1,300 \mathrm{~nm}$, the so-called diagnostic window, absorption is relatively low, so that light penetrates deep into the skin and optical inhomogeneities are the main factor influencing the image. OCT provides two-dimensional cross-sectional images of the skin (Fig. 2). The axial resolution depends on the coherence length of the light source, whereas the lateral resolution is given by the focal spot size and the scan step. Both values are about $15 \mathrm{~mm}$ for conventional OCT systems. The detection depth depends on the wavelength, the scattering and the attenuation of the light inside the tissue and varies from 1 to $1.5 \mathrm{~mm}$ in skin. ${ }^{3.4}$

\section{APPEARANCE OF NORMAL SKIN ON OCT}

To interprete OCT images of pathological processes, appearance of healthy skin has to be studied at first. A calculation of the thickness of layers, the intensity of the signal and the light attenuation coefficient in different depths can be performed in a region of interest in the OCT image. The reflectivity of the skin leads to a signal intense bright band and show peak on the surface, which is also considered the entrance signal. Below this signal layers and structures can be distinguished in the OCT image.

\section{Stratum coreneum}

Normally, the stratum corneum is only visible on palmoplantar skin (Fig. 3). At these sites, the horny layer of palmoplantar skin is a well-defined, thick, homogenous, low-scattering, well defined superficial layer with some 


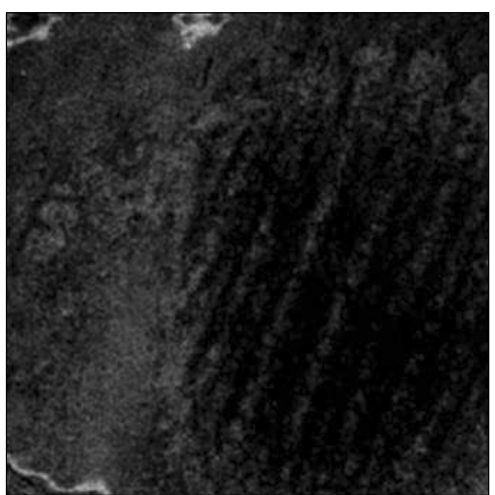

Fn-face image

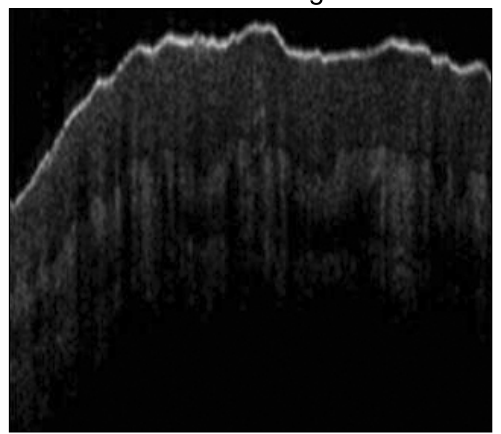

Cross-sectional image

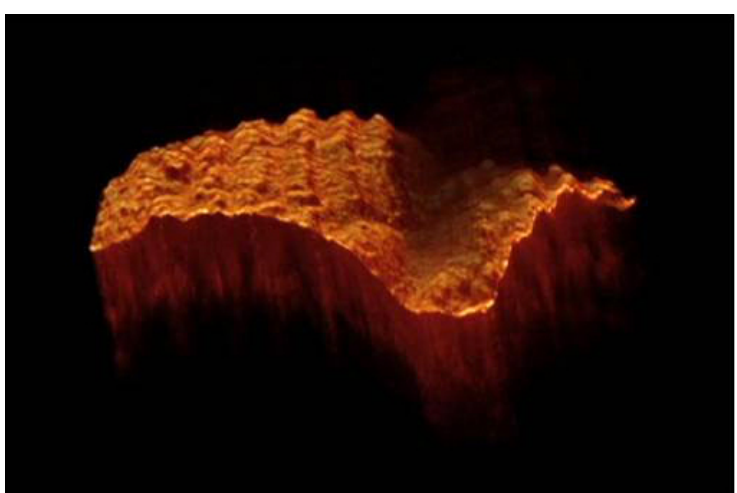

3D reconstruct (movie)

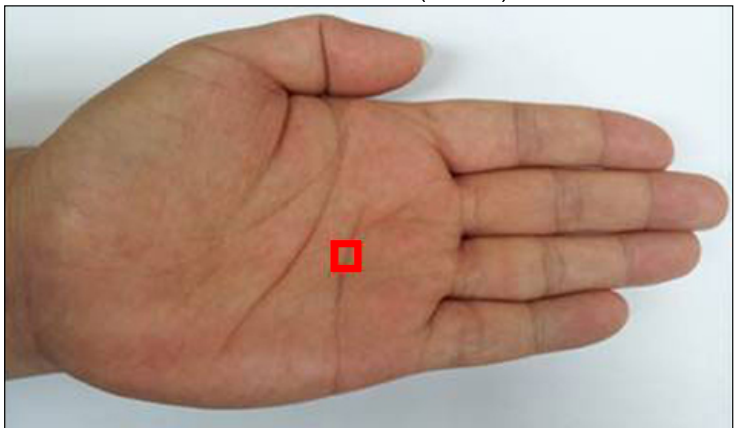

Fig. 2. OCT provides three \& twodimensional cross-sectional images of the skin.

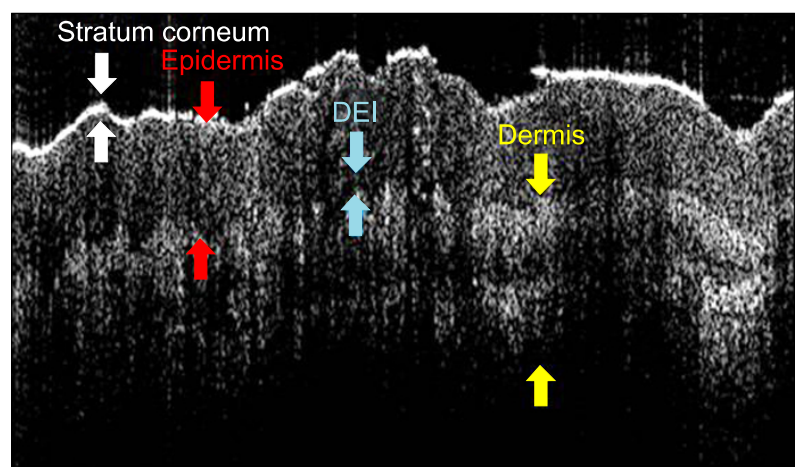

Fig. 3. OCT image on palmoplantar skin: Stratum corneum is visible. The horny layer of palmoplantar skin is a well-defined, thick, homogenous, low-scattering, well defined superficial layer with some spiral strong scattering sweat gland ducts inside.

spiral strong scattering sweat gland ducts inside.

The border between the cornified and living epidermis is usually distinct, whereas the dermo-epidermal border is frequently blurred. For example, the average thickness of the stratum corneum on the fingertips is about $300 \mathrm{~mm}$ by OCT in vivo. The thickness of the corresponding viable epidermis is about $100 \mathrm{~mm}$.

\section{Epidermis}

Except for palms and soles, the epidermis of the face and body is the first distinguishable layer in the OCT image. Stratum corneum is normally not thick enough to be visible at the resolution of conventional OCT technique. The epidermis shows less intense signals than the dermis and can be distinguished from the upper dermis if the border is flat and not too ridged. The most commonly used method for the measuring epidermal thickness can be easily be evaluated using the averaged A-scan. Distance between the first intensity peak lalso called entrance peak) and the second intensity peak in the averaged A-Scan most probably reflects epidermis thickness, because the valley before the second peak most likely corresponds to the dermo-epidermal junction.

The second reflecting layer, which causes the second peak in the A-scan, can be ascribed to backscattering of light at the fibrous structure of collagen in the dermis. Hence, peak-to-peak measurements probably overestimate epidermal thickness as recently demonstrated. Epidermal thickness as measured with OCT was found to decrease with age, independent of gender, body localization and skin-type. Only forehead skin was shown to be significantly thinner in old females when compared with that in old men. Recently, Josse et al. ${ }^{5}$ reported on a new automatic image processing method for measuring epidermal thickness from OCT images that significantly reduces calculation. The data 
can be analyzed more rapidly and reproducibly in clinical trials.

\section{Dermis}

The dermis is usually more signal intense with some signal-poor cavities corresponding to blood vessels and hair follicles, sebaceous glands. Hairs on the skin surface cause signal shadows. The blood vessels appear as signal-free round or longish structures. If the dermis is thin, the low reflecting subcutis is visible in the lower parts of the image. Dermal signal intensity on OCT can predominantly be ascribed to the extracellular matrix, in particular the collagen fibre network.

\section{Skin appendages}

Hair follicles and ducts of sebaceous glands can be seen as lower reflecting regions within the intensely reflecting epidermis.

\section{APPLICATIONS}

The clinical studies of OCT in dermatology revealed that the method is of value for diagnosis of some inflammatory and bullous skin diseases and for differential diagnosis of some tumors - for example, basal cell carcinoma, squamous cell carcinoma, melanocytic nevi and malignant melanoma. ${ }^{6}$ It is also value for diagnosis of infections, such as onychomycosis, larva migrans and scabies. Skin moisture and hydration can be monitored with OCT by determining the refractive index and scattering index, respectively. It was shown that the higher the abundance of water, the lower the refractive index. Skin atrophy induced by the use of a topical glucocorticosteroid was studied by Pagnoni et al. ${ }^{7}$ OCT also have been tested to monitor burn depth, tissue freezing and wound healing in animal or in vitro models.
Accordingly, OCT was used for monitoring the wound healing after laser thermal injury.

\section{CONCLUSION}

OCT at its present technical realization is a valuable non-invasive morphological method that is starting to become commercially available. Further developments will expand the possibilities and indications of OCT for clinical use.

\section{REFERENCES}

1. Welzel J. Optical coherence tomography in dermatology: a review. Skin Res Technol 2001;7:1-9.

2. Gambichler T, Jaedicke V, Terras S. Optical coherence tomography in dermatology: technical and clinical aspects. Arch Dermatol Res 2011;303:457-73.

3. Pierce MC, Strasswimmer J, Park BH, Cense B, de Boer JF. Advances in optical coherence tomography imaging for dermatology. J Invest Dermatol 2004;123:458-63.

4. Coulman SA, Birchall JC, Alex A, Pearton M, Hofer B, O'Mahony $C$, et al. In vivo, in situ imaging of microneedle insertion into the skin of human volunteers using optical coherence tomography. Pharm Res 2011;28:66-81.

5. Josse G, George J, Black D. Automatic measurement of epidermal thickness from optical coherence tomography images using a new algorithm. Skin Res Technol 2011;17:3149.

6. Gambichler T, Moussa G, Sand M, Sand D, Altmeyer P, Hoffmann K. Applications of optical coherence tomography in dermatology. J Dermatol Sci 2005;40:85-94.

7. Pagnoni A, Knuttel A, Welker P, Rist M, Stoudemayer T, Kolbe L, et al. Optical coherence tomography in dermatology. Skin Res Technol. 1999;5:83-7. 